\title{
Prevalence, Species Distribution and Antifungal Susceptibility Profile of Candida Species Isolated from Bloodstream of Critical Care Unit Patients in a Tertiary Care Hospital in Kenya
}

\author{
Danait Andemichael Solomon ${ }^{*}$, Andrew K. Nyerere${ }^{1}$, Alice Kanyua ${ }^{2}$, Caroline Wangari Ngugi ${ }^{1}$ \\ ${ }^{1}$ Department of Medical Microbiology, Jomo Kenyatta University of Agriculture and Technology, 62000-00200, Nairobi, Kenya \\ ${ }^{2}$ Nairobi Hospital, 30026-00100, Nairobi, Kenya \\ Email: *paunyt16@gmail.com
}

How to cite this paper: Solomon, D.A. Nyerere, A.K., Kanyua, A. and Ngugi, C.W. (2021) Prevalence, Species Distribution and Antifungal Susceptibility Profile of Candida Species Isolated from Bloodstream of Critical Care Unit Patients in a Tertiary Care Hospital in Kenya. Open Journal of Medical Microbiology, 11, 32-46. https://doi.org/10.4236/ojmm.2021.111003

Received: January 30, 2021

Accepted: March 8, 2021

Published: March 11, 2021

Copyright ( 2021 by author(s) and Scientific Research Publishing Inc. This work is licensed under the Creative Commons Attribution International License (CC BY 4.0).

http://creativecommons.org/licenses/by/4.0/ c) (i) Open Access

\begin{abstract}
The upsurge of candidemia in the past years has been an immense encumbrance on public health and the number of deaths caused by candidemia particularly in critical care unit patients is devastating. Candida species harbor a $30 \%-60 \%$ mortality rate and compared to stable people or those with less serious illnesses, this ranges from $60 \%$ to $80 \%$ of those who are chronically ill patients. Grounded on a recent report from a tertiary care hospital in Kenya showing the emergence of previously unobserved species: Candida auris, this study aimed to determine the prevalence, species distribution, and antifungal susceptibility profile of candidemia in critical care unit patients of the hospital. 378 Critical Care Unit patients were enrolled for the study from January 2019 to January 2020. Positive archived isolates were sub-cultured using Saboraud Dextrose Agar. Candida species were identified utilizing API20C AUX and Vitek-2. Antifungal susceptibility testing was conducted using the Liofilchem MIC Test strip. Out of 378 patients, thirty-one presented a positive culture for Candida species. The prevalence of Candidemia was $8.2 \%$ with 9 (29.03\%) Candida auris, 8 (25.81\%) Candida albicans, 6 (19.35\%) Candida parapsilosis, 3 (9.68\%) Candida famata, 3 (9.68\%) Candida tropicalis, 1 (3.23\%) Candida duobushaemolumonii, and 1 (3.23\%) Candida lusitaniae. A resistance pattern to Fluconazole was observed among Candida auris and Candida parapsilosis, and resistance to Flucytosine was observed in Candida tropicalis, whereas susceptible MIC values were obtained for the other drugs. There is an increase in candidemia among critical care unit patients in the health facility posing a public health challenge. Moreover, the onset of new species Candida auris which is unprecedented in Kenya warrants enhanced infection control, and the uniform resistance of Candida auris, Candida pa-
\end{abstract}


rapsilosis, and Candida tropicalis towards Fluconazole and Flucytosine necessitate constant drug monitoring for empirical treatment regime. In contrast, the high potency of Echinocandins and Amphotericin-B demonstrate them as the drug of choice.

\section{Keywords}

Critical Care Unit, Candida Species, Candida auris, Candidemia, Antifungal Susceptibility

\section{Introduction}

Fungal infections, explicitly Candida species have been the major cause of mortality and morbidity in hospitalized patients and predominantly in the Critical Care Units [1] [2]. Candida species reside inside the host as normal flora and dwell in the oral cavity of healthy human beings, however, in immunocompromised patients, these commensal microorganisms are capable of causing disease [3]. Prominently causing nosocomial infections, Candida spp. rates the fourth in causing all bloodstream infections and third of bloodstream infections in critically ill patients [4]. Worldwide in tertiary care hospitals, those results attribute to mortality rates of $15 \% \pm 35 \%$ in adults and $10 \% \pm 15 \%$ in neonates [5]. Based on studies done in several hospitalized patients, including intensive care unit patients, candidemia causes mortality of $47 \%$ among the patients [6].

In the USA, Candida species are considered as the third or fourth most causative agent of healthcare-acquired infections [5] and lead to a 30\%-60\% mortality rate of hospitalized patients [6]. These are associated with changes in the individuals' physiology and immunocompromised state which results in severe infections [3]. Several risk factors of the origin of the Candida infections in hospitalized patients could be endogenously brought by the patients themselves or could be from instruments in the hospital. It might also be contributed to contaminations of hospital surroundings or cross-infection from health workers which would attribute to the exogenous cause [5] [7] [8]. Candidemia is frequently seen among those infections, especially with patients in Critical Care Units [5]. Moreover, as a result of these infections, expanded costs in healthcare are incurred, ranging from $\$ 35,000$ to $\$ 68,000$ for a single candidemia case in the United States [8].

Patterns of anti-fungal drug resistance by Candida species have been causing stern public health challenges and were encompassed in CDC's 2013 Antibiotic Resistance Threat Report [9] [10]. Critically ill patients undergo invasive treatments and consume several anti-fungal drugs, however, results of anti-fungal resistance have been causing epidemiological unsustainability [11] [12]. Certain Candida strains are progressively resistant to commonly used antifungal drugs. Based on recent data from CDC, a discernable shift is observed in candidemia occurrences with augmented drug resistance to first-line and second-line anti- 
fungal drugs such as Azoles and Echinocandins [10]. In the United States, 46,000 hospitalized patients face Candida infections each year, and approximately 30\% of those who harbor drug-resistant Candida species are estimated to die during hospitalization [10]. Several reports state that the increase of anti-fungal resistance has been resulting in high mortality and morbidity in critical care patients [2] [13]. This has contributed to many deaths due to Candida infections in the past years [11] [12].

According to literature from Kenya, the significant agent for hospital infections is considered to be Candida albicans [14] [15]. However, Candida auris has recently been observed frequently in a tertiary care hospital in Nairobi, Kenya grounded on several lab reports from ICU and HDU patients. Swift dissemination of this multi-drug resistant species is discerned in different parts of the world [2]. It was first reported in 2009 and since then it has been detected in five continents causing serious hospital-acquired infections [2]. A study conducted in Kenya recorded a drug resistance pattern of Candida albicans and Candida parapsilosis isolates towards fluconazole [14] [16], however, drug susceptibility patterns of the novel species, Candida auris remains unreported up-to-date in Kenya. Therefore, the aim of this study was to determine the prevalence and antifungal susceptibility profile of Candida species among critical care unit patients of the hospital.

\section{Materials and Methods}

\subsection{Study Area}

The study was conducted in a tertiary care hospital in Nairobi, Kenya. The hospital has been officially operational since 9 April 1954 and is located on Argwings Kodhek Road, in the neighborhood of Upper Hill, in Kenya's capital city of Nairobi. The hospital is a high capacity hospital and serves different patients from the whole country [17].

\subsection{Study Population}

The study participants were enrolled from the intensive care unit and highly dependent unit of the hospital. The study population included patients of all age groups who had been requested for blood culture by a physician. A total of 378 patients based on Naing et al. (2006) [18] formula were tested from January 2019 to January 2020 for Candida bloodstream infection in ICU and HDU of Nairobi Hospital. Out of those thirty-one (31), patients showed positive culture for Candida and the isolates were archived.

$$
n=\frac{Z^{2}(P)(1-P)}{d^{2}}
$$

where: $n=$ Sample size.

$Z=$ The confidence interval at $95 \%$ (1.96).

$P=$ Estimate of the proportion or anticipated prevalence used $12.42 \%$ candidiasis from a population-based study in Africa [19]. 
$d=$ margin of error at $5 \%(0.05)$.

$$
n=\frac{Z^{2}(P)(1-P)}{d^{2}}=\frac{1.96^{2}(0.1242)(1-0.1242)}{0.05^{2}}=167
$$

\subsection{Culture of Isolates}

The archived isolates which had positive blood culture underwent a sub culturing method using Saboraud dextrose agar (SDA). Isolates that were stored in glycerol were inoculated in a Petri dish plate with a Saboraud Dextrose media and they were incubated in $37^{\circ} \mathrm{C}$ for $24-48$ hours. The appearance of creamy white colonies with soft texture was considered positive for Candida growth.

\subsection{API 20C AUX and VITEK-2 Tests}

A confirmatory test was done for the cultured isolates to identify the specific species using API 20C AUX (Biomerieux, USA). Isolated Candida colonies were inoculated in the API $20 \mathrm{C}$ cupules for positive or negative growth check in the 19 assimilation tests [20]. Incubation of the strip was done for 48 and 72 hours in $31^{\circ} \mathrm{C}$, after which the reactions were compared to the first cupule containing negative control. The numbers recorded were interpreted using apiweb ${ }^{\mathrm{TM}}$ software (Biomerieux, SA). Isolates that were identified as Candida famata by API 20C AUX (Biomerieux, USA) were confirmed using VITEK-2 version 8.1 (Biomerieux, USA) to differentiate whether they were truly Candida famata or they were misidentified Candida auris or Candida duobushaemolumonii. The test was done following the manufacturer's instructions for VITEK-2 (Biomerieux, USA) [21].

\subsection{Antifungal Susceptibility Testing}

Anti-fungal drug susceptibility was performed using Liofilchem MIC test strip (Liofilchem S.R.I., Italy) for Fluconazole, Micafungin, Caspofungin, Amphotericin-B, Flucytosine, and Voriconazole [22]. The Liofilchem MIC test strip (Liofilchem S.R.I., Italy) is a non-penetrating plastic material designed to have 15 two-fold dilutions of antibiotic concentrations [22]. The strip was placed in a Saboraud Dextrose Agar (SDA) inoculated with 0.5 McFarland pure colonies' suspension of the isolated species. Subsequently, the agar plates were incubated at $37^{\circ} \mathrm{C}$ and observed for Minimum Inhibitory Concentration (MIC) reading after 24 hours. The interpretation was done classifying the organisms as sensitive or resistant based on CLSI MA-27 guidelines [23].

Quality control of the Identification and Antifungal Susceptibility tests in this study was maintained using reference strains of Candida albicans ATCC: 14053 and Candida parapsilosis ATCC 22019.

\subsection{Data Analysis}

Data collected were documented in Microsoft Excel (Microsoft Corp, USA) before transferring to SPSS version 20 (IBM, USA) for analysis. Descriptive Statis- 
tics was done to determine the frequencies of the species distribution. Cross Tabulation was used to determine the Prevalence of Candida species in relation to age, ward, and gender. Chi-square was used for the level of significance. T-test was used to calculate the p-value association between HDU and ICU based on age, gender, and prevalence. Minimum and maximum susceptibility values were calculated for all the drugs tested to obtain the MIC range.

\section{Results}

\subsection{Characteristics of Study Population}

A total of 378 patients were tested from January 2019 to January 2020. The study population was patients admitted to ICU and HDU of the tertiary care hospital. Out of 378 patients, 203 were from ICU and 105 from HDU. The median age for ICU was 55 years ( 2 mon-95 years) and 61 years (16 - 101 years) in HDU with a p-value of 0.0534 (Table 1). The majority of the patients were males at 158 (57.9\%) and 60 (57.1\%), while females were 115 (42.1\%) and 45 (42.9\%) in ICU and HDU respectively. The prevalence of Candida infections was more prominent in ICU with a total of $24(77.4 \%)$ positive cases, with HDU having a lower prevalence of $7(22.6 \%)$ positive cases.

\subsection{Prevalence and Distribution of Candida Species}

Out of the 378 patients tested thirty-one (31) patients were found to have candidemia comprising $8.2 \%$ of the total patients. The highest prevalence was Candida auris at $29.03 \%$ and the lowest prevalence in Candida duobushaemolumonii and Candida lusitaniae, at $3.23 \%$ each (Figure 1).

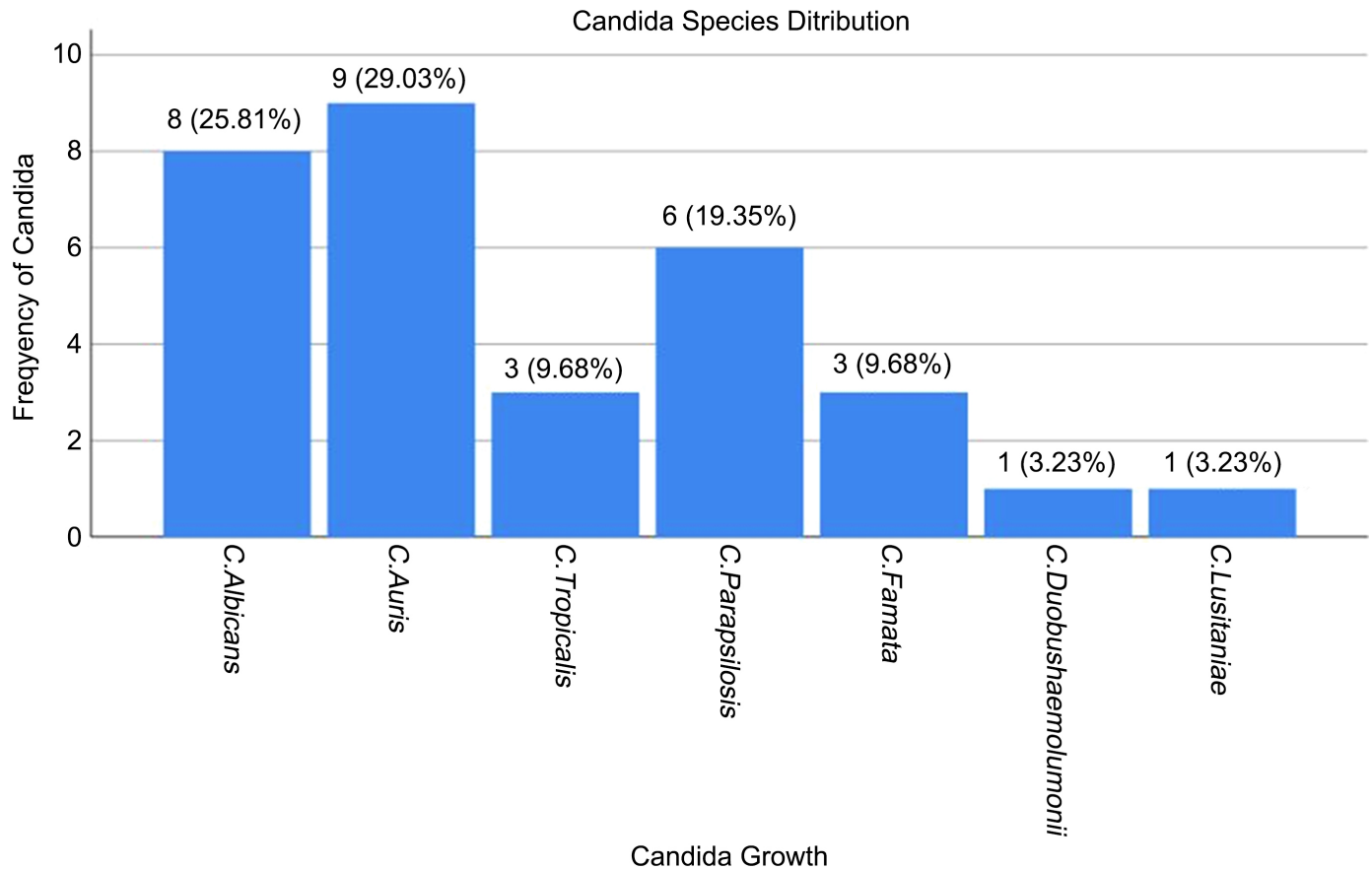

Figure 1. Distribution of Candida species from the API 20C AUX result. 
An overall higher prevalence was observed in ICU compared with HDU. In patients under the age of 20 years, there were two positive cases in male patients of HDU. In patients between the age of 20 - 40, there were four positive cases, three being among the male patients and 1 in the female patient of ICU. A total of seven patients (male and female) were positive for Candida species in ICU in the age group of $41-60$ while only two male patients were positive in HDU. The most noteworthy prevalence was seen in patients above the age of 60 with females comprising higher incidences of candidemia at $29.2 \%$ (7) while male patients had lesser prevalence at 25\% (6) in ICU. In contrast, males had a higher occurrence at $28.5 \%$ (2) than females at $14.3 \%$ (1) in HDU (Table 2). A chi-square test on both sides showed there was no significant association of the prevalence results with age, gender, and ward giving a p-value of $0.543,0.401$ and 0.436 , respectively.

Table 1. Characteristics of the study population.

\begin{tabular}{ccccc}
\hline & & ICU & HDU & P-VALUE \\
\hline Age & & & & \\
Median (Range) & M & 55 years (2 months - 95 years) & 61 years (16 years - 101 years) & 0.0534 \\
Gender & F & 158 & 60 & 0.908 \\
Prevalence (\%) & & 115 & 45 & 0.319 \\
\hline
\end{tabular}

Table 2. Distribution of Candida species based on age, ward, and gender.

\begin{tabular}{|c|c|c|c|c|c|c|c|c|}
\hline \multicolumn{9}{|c|}{ ICU } \\
\hline \multirow{2}{*}{$\begin{array}{c}\text { Age Group (Years) } \\
\text { Gender }\end{array}$} & \multicolumn{2}{|c|}{$<20$} & \multicolumn{2}{|c|}{$20-40$} & \multicolumn{2}{|c|}{$41-60$} & \multicolumn{2}{|c|}{$>60$} \\
\hline & $\mathbf{M}$ & F & $\mathbf{M}$ & F & $\mathbf{M}$ & F & $\mathbf{M}$ & F \\
\hline \multicolumn{9}{|l|}{ Organisms } \\
\hline Candida auris & $0(0 \%)$ & $0(0 \%)$ & $1(4.2 \%)$ & $1(4.2 \%)$ & $0(0 \%)$ & $0(0 \%)$ & $0(0 \%)$ & $4(16.6 \%)$ \\
\hline Candida albicans & $0(0 \%)$ & $0(0 \%)$ & $1(4.2 \%)$ & $0(0 \%)$ & $0(0 \%)$ & $2(8.3 \%)$ & $2(8.3 \%)$ & $2(8.3 \%)$ \\
\hline Candida parapsilosis & $0(0 \%)$ & $0(0 \%)$ & $1(4.2 \%)$ & $0(0 \%)$ & $2(8.3 \%)$ & $0(0 \%)$ & $2(8.3 \%)$ & $0(0 \%)$ \\
\hline Candida tropicalis & $0(0 \%)$ & $0(0 \%)$ & $0(0 \%)$ & $0(0 \%)$ & $0(0 \%)$ & $0(0 \%)$ & $1(4.2 \%)$ & $1(4.2 \%)$ \\
\hline Candida famata & $0(0 \%)$ & $0(0 \%)$ & $0(0 \%)$ & $0(0 \%)$ & $2(8.3 \%)$ & $0(0 \%)$ & $1(4.2 \%)$ & $0(0 \%)$ \\
\hline Candida duobushaemolumonii & $0(0 \%)$ & $0(0 \%)$ & $0(0 \%)$ & $0(0 \%)$ & $0(0 \%)$ & $0(0 \%)$ & $0(0 \%)$ & $0(0 \%)$ \\
\hline Candida lusitaniae & $0(0 \%)$ & $0(0 \%)$ & $0(0 \%)$ & $0(0 \%)$ & $0(0 \%)$ & $1(4.2 \%)$ & $0(0 \%)$ & $0(0 \%)$ \\
\hline \multicolumn{9}{|c|}{ HDU } \\
\hline Age Group (Years) & \multicolumn{2}{|c|}{$<20$} & \multicolumn{2}{|c|}{$20-40$} & \multicolumn{2}{|c|}{$41-60$} & \multicolumn{2}{|c|}{$>60$} \\
\hline Gender & $\mathbf{M}$ & F & $\mathbf{M}$ & F & $\mathbf{M}$ & F & $\mathbf{M}$ & F \\
\hline \multicolumn{9}{|l|}{ Organisms } \\
\hline Candida auris & $1(14.3 \%)$ & $0(0 \%)$ & $0(0 \%)$ & $0(0 \%)$ & $1(14.3 \%)$ & $0(0 \%)$ & $1(14.3 \%)$ & $0(0 \%)$ \\
\hline Candida albicans & $0(0 \%)$ & $0(0 \%)$ & $0(0 \%)$ & $0(0 \%)$ & $0(0 \%)$ & $0(0 \%)$ & $1(14.3 \%)$ & $0(0 \%)$ \\
\hline Candida parapsilosis & $0(0 \%)$ & $0(0 \%)$ & $0(0 \%)$ & $0(0 \%)$ & $0(0 \%)$ & $0(0 \%)$ & $0(0 \%)$ & $1(14.3 \%)$ \\
\hline Candida tropicalis & $0(0 \%)$ & $0(0 \%)$ & $0(0 \%)$ & $0(0 \%)$ & $1(14.3 \%)$ & $0(0 \%)$ & $0(0 \%)$ & $0(0 \%)$ \\
\hline Candida famata & $0(0 \%)$ & $0(0 \%)$ & $0(0 \%)$ & $0(0 \%)$ & $0(0 \%)$ & $0(0 \%)$ & $0(0 \%)$ & $0(0 \%)$ \\
\hline Candida duobushaemolumonii & $1(14.3 \%)$ & $0(0 \%)$ & $0(0 \%)$ & $0(0 \%)$ & $0(0 \%)$ & $0(0 \%)$ & $0(0 \%)$ & $0(0 \%)$ \\
\hline Candida lusitaniae & $0(0 \%)$ & $0(0 \%)$ & $0(0 \%)$ & $0(0 \%)$ & $0(0 \%)$ & $0(0 \%)$ & $0(0 \%)$ & $0(0 \%)$ \\
\hline
\end{tabular}




\subsection{Antifungal Susceptibility of Isolated Candida Species}

Antifungal Drug Susceptibility tests were accomplished for the 31 isolates against Fluconazole, Voriconazole, Micafungin, Caspofungin, Amphotericin B, and Flucytosine covering the three-drug classes of anti-fungal drugs. All Candida isolates in the study were sensitive to Micafungin, Caspofungin, Voriconazole, and Amphotericin B. However, Candida auris and Candida parapsilosis demonstrated complete resistance to Fluconazole whereas Candida tropicalis strains were resistant to Flucytosine (Table 3). The lowest MIC value was observed in Echinocandins, Micafungin at $0.023 \mu \mathrm{g} / \mathrm{mL}$ for Candida famata. Meanwhile, the highest MIC value obtained was in fluconazole resistance at $256 \mu \mathrm{g} / \mathrm{mL}$ and was observed in Candida auris and Candida parapsilosis strains. The test was conducted utilizing the Liofilchem MIC test strip and was interpreted based on the classification of CLSI breakpoints [23].

\section{Discussion}

This study documents the prevalence of candidemia among patients admitted to the critical care unit of a tertiary care hospital in Nairobi, Kenya. The results showed a higher prevalence of $8.2 \%$ compared to a study previously conducted in Kenya showing a rate of 5 cases of candidemia per 100,000 patients [15]. The increased candidemia could be attributed to the number of invasive measures taken in hospitalized patients when handling their medical care and the immunocompromised state of the patients also plays a role in the severity of the infection. This ascent in prevalence demonstrates a public health challenge, especially to those who are hospitalized in ICU and HDU and have relatively severe illnesses.

Existing publications of Candida infections in Kenya states that the most frequently isolated Candida species was Candida albicans [14] [16], however, this study highlights the onset of the new species, Candida auris in high prevalence. Candida auris was first isolated in 2009 and recorded cases are portrayed by elevated levels of total mortality [2] [24] [25] and high antifungal resistance rates [26]. Of note, the vast majority of the infections reported involved patients that are critically ill [24] [27]. Furthermore, identification has been difficult by microbiological techniques [2] [28] [29] notwithstanding Candida auris being one of the most prevalent species which increases the risk of misidentification and hence improper treatment. Candida auris's high virulence [30] [31], a profile of multi-drug resistance [32], and rapid outbreak and global propagation marks it as a worldwide threat [2].

The antifungal susceptibility tests performed showed a susceptible profile of all isolates towards Echinocandins and Amphotericin B and Voriconazole. A study conducted in a tertiary care hospital in Italy, however, showed a resistance pattern of Candida albicans towards Voriconazole [33]. Besides, as evidenced in certain reports Candida species are usually susceptible to Amphotericin-B as an antifungal drug [33], and the resistance to Echinocandins is in very low percentages 
Table 3. Anti-fungal susceptibility results and minimum inhibitory concentration (MIC) range against six antifungal drugs as evaluated by the Liofilchem MIC Test strip and distribution of isolates of Candida spp.

\begin{tabular}{|c|c|c|c|c|}
\hline \multirow[b]{2}{*}{ Candida Species } & \multicolumn{4}{|c|}{ No. (\%) Sensitivity Profile } \\
\hline & Total (\%) & $\begin{array}{l}\text { MIC Range } \\
(\mu \mathrm{g} / \mathrm{mL})\end{array}$ & Sensitive (\%) & Resistant (\%) \\
\hline Candida auris & $9(29.03 \%)$ & & & \\
\hline Fluconazole & & $>64-256$ & 0 & $9(100 \%)$ \\
\hline Voriconazole & & $0.19-1.5$ & $9(100 \%)$ & 0 \\
\hline Caspofungin & & $0.19-1$ & $9(100 \%)$ & 0 \\
\hline Micafungin & & $0.06-1$ & $9(100 \%)$ & 0 \\
\hline Amphotericin-B & & $0.064-0.75$ & $9(100 \%)$ & 0 \\
\hline Flucytosine & & $0.50-1$ & $9(100 \%)$ & 0 \\
\hline Candida albicans & $8(25.81 \%)$ & & & \\
\hline Fluconazole & & $0.5-4$ & $8(100 \%)$ & 0 \\
\hline Voriconazole & & $0.125-1$ & $8(100 \%)$ & 0 \\
\hline Caspofungin & & $0.012-0.94$ & $8(100 \%)$ & 0 \\
\hline Micafungin & & $0.06-1$ & $8(100 \%)$ & 0 \\
\hline Amphotericin-B & & $0.25-0.50$ & $8(100 \%)$ & 0 \\
\hline Flucytosine & & $1-1.5$ & $8(100 \%)$ & 0 \\
\hline Candida tropicalis & $3(9.68 \%)$ & & & \\
\hline Fluconazole & & $1-1$ & $3(100 \%)$ & 0 \\
\hline Voriconazole & & $0.125-0.75$ & $3(100 \%)$ & 0 \\
\hline Caspofungin & & $0.016-0.25$ & $3(100 \%)$ & 0 \\
\hline Micafungin & & $0.50-0.75$ & $3(100 \%)$ & 0 \\
\hline Amphotericin-B & & $0.38-0.50$ & $3(100 \%)$ & 0 \\
\hline Flucytosine & & $>32$ & 0 & $3(100 \%)$ \\
\hline Candida famata & $3(9.68 \%)$ & & & \\
\hline Fluconazole & & $0.50-1$ & $3(100 \%)$ & 0 \\
\hline Voriconazole & & $0.094-1$ & $3(100 \%)$ & 0 \\
\hline Caspofungin & & $0.25-1$ & $3(100 \%)$ & 0 \\
\hline Micafungin & & $0.023-0.38$ & $3(100 \%)$ & 0 \\
\hline Amphotericin-B & & $0.047-0.5$ & $3(100 \%)$ & 0 \\
\hline Flucytosine & & $0.38-1$ & $3(100 \%)$ & 0 \\
\hline Candida parapsilosis & $6(19.35 \%)$ & & & \\
\hline Fluconazole & & $>64-256$ & 0 & $6(100 \%)$ \\
\hline Voriconazole & & $0.047-1$ & $6(100 \%)$ & 0 \\
\hline Caspofungin & & $0.50-1$ & $6(100 \%)$ & 0 \\
\hline Micafungin & & $0.25-1$ & $6(100 \%)$ & 0 \\
\hline Amphotericin-B & & $0.25-1$ & $6(100 \%)$ & 0 \\
\hline Flucytosine & & $0.75-1$ & $6(100 \%)$ & 0 \\
\hline
\end{tabular}




\section{Continued}

\begin{tabular}{|c|c|c|c|c|}
\hline Candida Lusitaniae & $1(3.23 \%)$ & & & \\
\hline Fluconazole & & - & $1(100 \%)$ & 0 \\
\hline Voriconazole & & - & $1(100 \%)$ & 0 \\
\hline Caspofungin & & - & $1(100 \%)$ & 0 \\
\hline Micafungin & & - & $1(100 \%)$ & 0 \\
\hline Amphotericin-B & & - & $1(100 \%)$ & 0 \\
\hline Flucytosine & & - & $1(100 \%)$ & 0 \\
\hline Candida Duobushaemolumonii & $1(3.23 \%)$ & & & \\
\hline Fluconazole & & - & $1(100 \%)$ & 0 \\
\hline Voriconazole & & - & $1(100 \%)$ & 0 \\
\hline Caspofungin & & - & $1(100 \%)$ & 0 \\
\hline Micafungin & & - & $1(100 \%)$ & 0 \\
\hline Amphotericin-B & & - & $1(100 \%)$ & 0 \\
\hline Flucytosine & & - & $1(100 \%)$ & 0 \\
\hline
\end{tabular}

Classification of sensitive and resistant according to the rules of the Clinical and Laboratory Standards Institute M27-A3 [23]: Fluconazole (S: $8 \mu \mathrm{g} / \mathrm{mL} ; \mathrm{R}: 64 \mu \mathrm{g} / \mathrm{ml}$ ); Voriconazole (S: $1 \mu \mathrm{g} / \mathrm{mL} ; \mathrm{R}: 4 \mu \mathrm{g} / \mathrm{mL}$ ); Flucytosine (S: $4 \mu \mathrm{g} / \mathrm{mL} ; \mathrm{R}: 32 \mu \mathrm{g} / \mathrm{mL}$ ); Amphotericin B (S $<1 \mu \mathrm{g} / \mathrm{mL} ; \mathrm{R}>2 \mu \mathrm{g} / \mathrm{mL}$ ); Echinocandins $(\mathrm{S}<2 \mu \mathrm{g} / \mathrm{ml}$, sensitive breakpoint only). S, sensitive; R, resistant.

to Candida albicans and non-albicans except for Candida glabrata [34]. Moreover, a study conducted in Kenya showed a resistance pattern of Candida albicans towards Fluconazole at a rate of $26 \%$ [16]. Interestingly, there was no isolate of Candida albicans showing resistance to any of the drug classes used in this study. Aware of the latter assertion, the increased susceptibility of Candida albicans might indicate the enhancement of drug regime towards this species as research was done massively targeting it in Kenya [14] [15] [35].

Furthermore, an increased resistance pattern in three species was observed in this study. Candida auris was seen to have a high resistance pattern up to 256 $\mu \mathrm{g} / \mathrm{ml}$ for fluconazole indicating no susceptible isolate from all the 9 isolates tested against fluconazole. The power of Candida auris to establish uniform resistance to fluconazole might increase the mortality rates of immunocompromised patients [2] [36]. This study additionally acquired a drug resistance pattern towards Flucytosine by Candida tropicalis with 3 (100\%) isolates showing increased MIC values as was classified resistant by CLSI breakpoints. A study conducted in Paris indicates the increased resistant activity of Candida tropicalis towards Flucytosine [37], however, this has not been documented in Kenya previously. In spite of certain reports expressing Candida parapsilosis as frequently susceptible to azoles [38] [39], several pieces of research have indicated its increased resistance towards Fluconazole [23] [40] [41]. In this study, all isolated Candida parapsilosis were discovered resistant to Fluconazole.

The drug susceptibility results exhibited a resistance pattern in the entirety of the isolated species of Candida parapsilosis, Candida auris, and Candida tropica- 
lis. Critically ill patients face the threat of morbidity and mortality as a result of the wide use of broad-spectrum anti-fungal drugs where there is a development of resistance by Candida species [2]. Inherent causes of resistance are also noted on top of the usual acquired pattern of drug resistance after antifungal drugs are administered to patients [2] [42]. This intrinsic nature of resistance is usually seen in non-albicans, as is documented in this study. Despite the fact that the onset of drug resistance happens in several cases the pattern of complete resistance in all three species might show transmission of the Candida species among the patients. Infection control in patients with Candida has never been a challenge previously since it was comprehended that Candida infections derive essentially from the translocation of host flora to usually sterile locations, such as the bloodstream. Similar patterns of resistance, however, may suggest that the same strain circulates across the patients attributed to hospitalized patients' transmission as is confirmed by numerous studies [43] [44] [45]. The increased resistance of the Candida species inclines a high risk to hospitalized patients and might attribute to increased death rate particularly in critically ill patients.

\section{Conclusion}

There are increased candidemia cases in the critical care units of the health facility and dependent on the outcomes acquired, the study determined an exceptionally pervasive occurrence of new Candida species: Candida auris, and its drug-resistant property as a high threat to the public. Thus, measures ought to be taken to control the infection and transmission to diminish the mortality and morbidity of critically ill patients. Additionally, Echinocandins showed high potency as antifungal drugs against albicans and non-albicans whereas Fluconazole and Flucytosine were proved to be less effective drugs in the conspicuously seen species of Candida auris, Candida parapsilosis, and Candida tropicalis. Consequently, for an appropriate drug regime, physicians should be alert of the drug susceptibility patterns of Candida species in the locality before prescribing any of the anti-fungal drugs. All in all, the onset of new species and increased drug resistance patterns warrant constant drug monitoring. As this study was limited to Nairobi, researches of Candida blood stream infections in critical care units should be done in hospitals in other parts of Kenya for a comprehensive and updated prevalence study and to monitor the spread of Candida auris in the country. Moreover, the molecular basis of resistance should also be established in future studies for enhanced treatment.

\section{Acknowledgements}

We would like to acknowledge AFRICA-ai-JAPAN Project for funding this research. We would also like to convey our gratitude to Nairobi Hospital, especially the Microbiology Department and Mrs. Felister Musyoki, and One health, HORN for their material and physical support. Our appreciation goes to Mr. Pawlos Semere Tsegay for his continuous support throughout the research and 
Mr. James Gachugia for his assistance in editing the manuscript.

\section{Funding}

This project is funded by AFRICA-ai-JAPAN Project for materials and reagents used in the research.

\section{Authors Contribution}

DAS, AKN, AK, CWN conceived and designed the study. DAS performed laboratory analysis and data analysis. DAS drafted the manuscript. All authors read, reviewed, and approved the final manuscript.

\section{Ethical Approval}

Ethical approval was obtained from Jomo Kenyatta University of Agriculture and Technology's Ethics Review Committee and the study site hospital's Ethics Review Committee. No patient was recruited solely for the study, but rather the study was part of the normal patient care process. Access to the data was strictly prohibited to maintain the confidential information of the patient. Patient Identification was done in a way that doesn't disclose the real information of the patient to avoid the leak of patient history.

\section{Data Availability}

The data can be provided upon request from the corresponding author.

\section{Conflict of Interest}

The authors declare that there is no conflict of interest regarding the publication of this paper.

\section{References}

[1] Kullberg, B.J. and Arendrup, M.C. (2015) Invasive Candidiasis. The New England Journal of Medicine, 373, 1445-1456.

[2] Cortegiani, A., Misseri, G., Fasciana, T., Giammanco, A., Giarratano, A. and Chowdhary, A. (2018) Epidemiology, Clinical Characteristics, Resistance, and Treatment of Infections by Candida auris. Journal of Intensive Care, 6, Article No. 69. https://doi.org/10.1186/s40560-018-0342-4

[3] Sardi, J.C.O., Duque, C., Hofling, J.F. and Goncalves, R.B. (2012) Genetic and Phenotypic Evaluation of Candida albicans Strains Isolated from Subgingival Biofilm of Diabetic Patients with Chronic Periodontitis. Medical Mycology, 50, 467-475. https://doi.org/10.3109/13693786.2011.633233

[4] Sandt, C., Sockalingum, G.D., Aubert, D., Lepan, H., Lepouse, C., Jaussaud, M., et al. (2003) Use of Fourier-Transform Infrared Spectroscopy for Typing of Candidaalbicans Strains Isolated in Intensive Care Units. Journal of Clinical Microbiology, 41, 954-959. https://doi.org/10.1128/JCM.41.3.954-959.2003

[5] Al-Obaid, K., Asadzadeh, M., Ahmad, S. and Khan, Z. (2017) Population Structure and Molecular Genetic Characterization of Clinical Candida tropicalis Isolates from 
a Tertiary-Care Hospital in Kuwait Reveal Infections with Unique Strains. PLoS ONE, 12, e0182292. https://doi.org/10.1371/journal.pone.0182292

[6] Bonfim-Mendonça, P.D.S., Fiorini, A., Shinobu-Mesquita, C.S., Baeza, L.C., Fernandez, M.A. and Svidzinski, T.I.E. (2013) Molecular Typing of Candida albicans Isolates from Hospitalized Patients. Revista do Instituto de Medicina Tropical de São Paulo, 55, 385-391. https://doi.org/10.1590/S0036-46652013000600003

[7] Ahmad, S. and Khan, Z. (2012) Invasive Candidiasis: A Review of Nonculture-Based Laboratory Diagnostic Methods. Indian Journal of Medical Microbiology, 30, 264-269. https://doi.org/10.4103/0255-0857.99482

[8] Suleyman, G. and Alangaden, G.J. (2016) Nosocomial Fungal Infections Epidemiology, Infection Control, and Prevention. Infectious Disease Clinics of North America, 30, 1023-1052. https://doi.org/10.1016/j.idc.2016.07.008

[9] Toda, M., Williams, S.R., Berkow, E.L., Farley, M.M., Harrison, L.H., Bonner, L., et al. (2019) Population-Based Active Surveillance for Culture-Confirmed Candidemia-Four Sites, United States, 2012-2016. Morbidity and Mortality Weekly Report-Surveillance Summaries, 68, 1-15. https://doi.org/10.15585/mmwr.ss6808a1

[10] Centers for Disease Control and Prevention (2013) Antibiotic Resistance Threats in the United States, 2013. US Centers for Disease Control and Prevention, Atlanta.

[11] Ghahri, M., Mirhendi, H., Zomorodian, K. and Kondori, N. (2013) Identification and Antifungal Susceptibility Patterns of Candida Strains Isolated from Blood Specimens in Iran. Archives of Clinical Infectious Diseases, 8, e14529. https://doi.org/10.5812/archcid.14529

[12] Sadrossadati, S.Z., Ghahri, M., Fooladi, A.A.I., Sayyahfar, S., Beyraghi, S. and Baseri, Z. (2018) Phenotypic and Genotypic Characterization of Candida Species Isolated from Candideamia in Iran. Current Medical Mycology, 4, 14-20. https://doi.org/10.18502/cmm.4.2.64

[13] Negri, M., Silva, S., Henriques, M. and Oliveira, R. (2012) Insights into Candida tropicalis Nosocomial Infections and Virulence Factors. European Journal of Clinical Microbiology \& Infectious Diseases, 31, 1399-1412. https://doi.org/10.1007/s10096-011-1455-Z

[14] Kangogo, M., Wanyoike, M., RevathiI, G. and Bii, C. (2011) Phenotypic Characterization of Candida albicans from Clinical Sources in Nairobi. African Journal of Health Sciences, 19, 19-23.

[15] Guto, J.A., Bii, C.C. and Denning, D.W. (2016) Emerging Problems in Infectious Diseases Estimated Burden of Fungal Infections in Kenya. Journal of Infection in Developing Country, 10, 777-784. https://doi.org/10.3855/jidc.7614

[16] Ooga, V.B., Gikunju, J.K. and Bii, C.C. (2011) Characterization and Antifungal Drug Susceptibility of Clinical Isolates of Candida Species. African Journal of Health Sciences, 19, 76-83.

[17] The Nairobi Hospital (2016) Annual Report \& Financial Statements. The Nairobi Hospital, Nairobi.

[18] Naing, L., Winn, T. and Rusli, B.N. (2006) Pratical Issues in Calculating the Sample Size for Prevalence Studies. Archives of Orofacial Sciences, 1, 9-14.

[19] Omrani, A.S., Pecen, L., Hajek, P., Raghubir, N. and Zigmond, J. (2014) Prevalence of Invasive and Superficial Candida Infections in Africa and Middle East: A Systematic Review and Meta-Analysis. 114th Meeting of the American Society for Microbiology, Boston, 17-20 May 2014, No. 1273.

[20] BioMérieux SA (2010) 20 C AUX. BioMérieux SA, Marcy-l’Etoile, 1-3. 
[21] Pincus, D.H. (2013) Microbial Identification Using the Biomerieux VITEK 2 System. In: Miller, M.J., Ed., Encyclopedia of Rapid Microbiological Methods, 1-32.

[22] Liofilchem (2018) Liofilchem MIC Test Strip. Liofilchem, Waltham.

[23] Cantón, E., Espinel-Ingroff, A. and Pemán, J. (2009) Trends in Antifungal Susceptibility Testing Using CLSI Reference and Commercial Methods. Expert Review of Anti-Infective Therapy, 7, 107-119. https://doi.org/10.1586/14787210.7.1.107

[24] Rudramurthy, S.M., Chakrabarti, A., Paul, R.A., Sood, P., Kaur, H., Capoor, M.R., et al. (2017) Candida auris Candidaemia in Indian ICUs: Analysis of Risk Factors. Journal of Antimicrobial Chemotherapy, 72, 1794-1801. https://doi.org/10.1093/jac/dkx034

[25] Sekyere, J.O. (2018) Candida auris. A Systematic Review and Meta-Analysis of Current Updates on an Emerging Multidrug-Resistant Pathogen. MicrobiologyOpen, 7, e00578.

[26] Ben-Ami, R., Berman, J., Novikov, A., Bash, E., Shachor-Meyouhas, Y., Zakin, S., Maor, Y., Tarabia, J., Schechner, V., Adler, A. and Finn, T. (2018) Multidrug-Resistant Candida haemulonii and C. auris, Tel Aviv, Israel. Emerging Infectious Diseases, 23, 195-203. https://doi.org/10.3201/eid2302.161486

[27] Chowdhary, A., Voss, A. and Meis, J.F. (2016) Multidrug-Resistant Candida auris. 'New Kid on the Block' in Hospital-Associated Infections? Journal of Hospital Infection, 94, 209-212. https://doi.org/10.1016/j.jhin.2016.08.004

[28] Kathuria, S., Singh, P.K., Sharma, C., Prakash, A., Masih, A., Kumar, A., et al. (2015) Multidrug-Resistant Candida auris Misidentified as Candida haemulonii: Characterization by Matrix-Assisted Laser Desorption Ionization-Time of Flight Mass Spectrometry and DNA Sequencing and Its Antifungal Susceptibility Profile Variability by Vitek 2, CLSI Broth Microdilution, and Etest Method. Journal of Clinical Microbiology, 53, 1823-1830. https://doi.org/10.1128/JCM.00367-15

[29] Kim, T.-H., Kweon, O.J., Kim, H.R. and Lee, M.-K. (2016) Identification of Uncommon Candida Species Using Commercial Identification Systems. Journal of Microbiolog and Biotechnology, 26, 2206-2213.

https://doi.org/10.4014/jmb.1609.09012

[30] Fakhim, H., Vaezi, A., Dannaoui, E., Chowdhary, A., Nasiry, D., Faeli, L., et al. (2018) Comparative Virulence of Candida auris with Candida haemulonii, Candida glabrata and Candida albicans in a Murine Model. Mycoses, 61, 377-382. https://doi.org/10.1111/myc. 12754

[31] Sarma, S. and Upadhyay, S. (2017) Current Perspective on Emergence, Diagnosis and Drug Resistance in Candida auris. Infection and Drug Resistance, 10, 155-165. https://doi.org/10.2147/IDR.S116229

[32] Sharma, C., Kumar, N., Pandey, R., Meis, J.F. and Chowdhary, A. (2016) Whole Genome Sequencing of Emerging Multidrug Resistant Candida auris Isolates in India Demonstrates Low Genetic Variation. New Microbes and New Infections, 13, 77-82. https://doi.org/10.1016/j.nmni.2016.07.003

[33] Bassetti, M., Taramasso, L., Nicco, E., Molinari, M.P., Mussap, M. and Viscoli, C. (2011) Epidemiology, Species Distribution, Antifungal Susceptibility and Outcome of Nosocomial Candidemia in a Tertiary Care Hospital in Italy. PLoS ONE, 6, e24198. https://doi.org/10.1371/journal.pone.0024198

[34] Perlin, D.S. (2015) Echinocandin Resistance in Candida. Clinical Infectious Diseases, 61, S612-S617. https://doi.org/10.1093/cid/civ791

[35] Bii, C.C., Kangogo, M., Revathi, G. and Wanyoike, W. (2009) Genotypes of Candida albicans from clinical sources in Nairobi Kenya. African Journal of Microbiology 
Research, 3, 475-477.

[36] Navalkele, B.D., Revankar, S. and Chandrasekar, P. (2017) Candida auris: A Worrisome, Globally Emerging Pathogen. Expert Review of Anti-Infective Therapy, 15, 819-827. https://doi.org/10.1080/14787210.2017.1364992

[37] Desnos-Ollivier, M., Bretagne, S., Bernède, C., Robert, V., Raoux, D., Elisabeth, C., Forget, E., Lacroix, C., Dromer, F. and the YEASTS Group (2008) Clonal Population of Flucytosine-Resistant Candida tropicalis from Blood Cultures, Paris, France. Emerging Infectious Diseases, 14, 557-565. https://doi.org/10.3201/eid1404.071083

[38] Lotfali, E., Kordbacheh, P., Mirhendi, H., Zaini, F., Ghajari, A., Mohammadi, R., et al. (2016) Antifungal Susceptibility Analysis of Clinical Isolates of Candida parapsilosis in Iran. Iranian Journal of Public Health, 45, 322-328.

[39] Eksi, F., Gayyurhan, E.D. and Balci, I. (2013) In Vitro Susceptibility of Candida Species to Four Antifungal Agents Assessed by the Reference Broth Microdilution Method. The Scientific World Journal, 2013, Article ID: 236903.

https://doi.org/10.1155/2013/236903

[40] Souza, A.C.R., Fuchs, B.B., Pinhati, H.M.S., Siqueira, R.A., Hagen, F., Meis, J.F., et al. (2015) Candida parapsilosis Resistance to Fluconazole: Molecular Mechanisms and In Vivo Impact in Infected Galleria mellonella Larvae. Antimicrobial Agents and Chemotherapy, 59, 6581-6587. https://doi.org/10.1128/AAC.01177-15

[41] Cleveland, A.A., Farley, M.M., Harrison, L.H., Stein, B., Hollick, R., Lockhart, S.R., et al. (2012) Changes in Incidence and Antifungal Drug Resistance in Candidemia: Results from Population-Based Laboratory Surveillance in Atlanta and Baltimore, 2008-2011. Clinical Infectious Diseases, 55, 1352-1361. https://doi.org/10.1093/cid/cis697

[42] Mandelblat, M., Frenkel, M., Abbey, D., Ben, R., Berman, J. and Segal, E. (2017) Phenotypic and Genotypic Characteristics of Candida albicans Isolates from Bloodstream and Mucosal Infections. Mycoses, 60, 534-545. https://doi.org/10.1111/myc. 12623

[43] Ture, Z. and Alp, E. (2018) Infection Control Measures to Prevent Hospital Transmission of Candida. Hospital Practice, 46, 253-257. https://doi.org/10.1080/21548331.2018.1510282

[44] Schwartz, I.S., Smith, S.W. and Dingle, T.C. (2018) Something Wicked This Way Comes: What Health Care Providers Need to Know about Candida auris. Canada Communicable Disease Report, 44, 271-276. https://doi.org/10.14745/ccdr.v44i11a01

[45] Schelenz, S., Hagen, F., Rhodes, J.L., Abdolrasouli, A., Chowdhary, A., Hall, A., et al. (2016) First Hospital Outbreak of the Globally Emerging Candida auris in a European Hospital. Antimicrobial Resistance \& Infection Control, 5, Article No. 35. https://doi.org/10.1186/s13756-016-0132-5 


\section{Abbreviations}

CCU: Critical Care Units;

ICU: Intensive Care Unit;

HDU: Highly Dependent Unit;

SDA: Saboraud Dextrose Agar;

JKUAT: Jomo Kenyatta University of Agriculture and Technology. 